\title{
The Orientation of Future JPL Planetary Ephemerides
}

\author{
E. M. Standish \\ JPL/Caltech; 901-150; Pasadena, CA 91109; USA; ems@smyles.jpl.nasa.gov
}

\begin{abstract}
In the future, the ephemerides produced at JPL will be based upon the reference frame of the radio source catalogues. Recent planetary observations have been made directly with respect to the radio frame, and these observations have shown a satisfying degree of absolute accuracy and internal consistency; they provide an automatic frame-tie.
\end{abstract}

\section{Orientations of Past Ephemerides at JPL}

In the past, the ephemerides of the inner planetary system, have been very well determined in a relative sense, due to the highly accurate ranging observations. However, of the earlier data types to which the ephemerides are adjusted, only the optical observations are referenced to an outside reference frame, and consequently, the earlier JPL ephemerides (up to and including DE130) were oriented onto the FK4 by the optical observations. Since these observations are two orders of magnitude less accurate than the other data types, the orientation of the ephemerides onto a celestial reference frame represented one of the least accurate features of the ephemerides. Starting with DE200, attempts were made to orient the ephemerides onto the mean equator and mean dynamical equinox of $J 2000$ [Standish, 1982. These attempts were not entirely successful, however, due to problems of defining the ecliptic, to determining the mean, and to using an inaccurate value of precession.

\section{Planetary Ephemeris - IERS Frame-Tie}

The VLBI observations in 1989 from the Phobos Spacecraft aproaching Mars and in 1992-4 from the Magellan Spacecraft orbiting Venus may be used to determine an offset between a given planetary ephemeris and the radio source (IERS) catalogue used for the observations. A rotation matrix is determined which re-orients the ephemeris in order to give a best-fit to the observations; that rotation matrix is the frame-tie.

An alternative method for determining the same frame-tie was used by Folkner et al., 1994. For DE200, the former and latter methods give, respectively,

$(-0 ! \cdot 002,-0 ! 003,-0 ! \cdot 002)[ \pm 0 ! 002]$ and $(-0 ! \cdot 002,-0 ! 012,-0 ! \cdot 006)[ \pm 0 ! 004]$.

Comparison shows good agreement between the two methods and, importantly, that DE200 is very close to the IERS reference frame, differing by only $0 ! " 01$.

\section{Conclusion: Future JPL Ephemerides}

The radio frame is accurate, stable, and well-defined; earth-orientation is now done with respect to the IERS frame; a significant number of VLBI observations of the inner planets with respect to the IERS frame have now being taken; and CCD measurements of the outer planets, their satellites, and QSO's now enable those objects to be tied onto the radio frame.

Future JPL ephemerides will be based upon the radio source reference frame, and since DE200 frame is so close to that of the IERS, the transformation onto the frame of the IERS will not represent a major change.

\section{References}

Folkner,W.M., Charlot,P., Finger,M.H., Williams,J.G., Sovers,O.J., Newhall, XX, \& Standish,E.M. (1994) Determination of the extragalactic frame tie from joint analysis of radio interferometric and lunar laser ranging measurements, Astron. Astrophys., 287, 279-289.

Standish,E.M. (1982) Orientation of the JPL Ephemerides, DE200/LE200, to the Dynamical Equinox of J2000, Astron. Astrophys., 114, 297-302. 